on recombinant DNA methodology. This one is based on a practical course run by the University of Leicester and contains five detailed articles: Mammalian cDNA cloning; Analysis of nucleic acids (restriction maps, Southern and Northern blots); Systems for the analysis of gene expression; Cloning in phage lambda; and electron microscopy of nucleic acids. Thus, it covers some areas that are not dealt with in other widely used texts such as that of Maniatis et al. The strength of this book is the large amount of practical detail that is included, especially useful for beginners in this field. Its coverage is limited to the five topics listed above and would therefore need to be supplemented by other articles. However, no attempt is made to guide the reader to any of this material, and in general the references given are rather few in number and not particularly up to date. For example, in the chapter on lambda cloning there is no mention of the recently developed and extremely useful EMBL series of vectors.

I would suggest that potential buyers have a good look at the book before they commit themselves, and be aware that more up to date procedures are available in many cases.

Duncan Shaw

\section{Gene Expression During Normal and Malignant Differentiation}

Edited by L C Andersson, C G Gahmberg, and P Ekblom. (Pp 237; figures + tables. £26.50.) Florida: Academic Press. 1985.

As described in the preface, this book comprises the highlights of a symposium held in 1984. The symposium was intended to cover the genetic regulation of cellular functions from diverse positions. The combination of an all-encompassing title and a limited number of contributions has selfevident inherent problems which have not been entirely overcome. To satisfy the bias of current interest in molecular biology, eight of the 18 chapters deal with the effects of viral and cellular oncogenes. These are overall fairly informative articles, dealing with oncogene activation, expression in normal and neoplastic cells, phosphorylation, similarity of oncogene products and growth factors, HTLV, and so forth, but their number tends somewhat to compress illustrations of other viewpoints. Representations of these include influence of cell-cell interactions and the architectural features of the cell ('Cells as tensegrity structures') on differentiation, various aspects of induction and control of differentiation, and patterns of membrane glycoproteins and glycolipids, associated with differentiation and proliferation.

It is highly unlikely that specialists will find anything in the relevant chapters with which they will be unfamiliar. Anything which fulfilled the criterion of 'very recent developments in biomedical research' at the time of the symposium has since been published in far more detail in the refereed journals. The book may be of interest to the specialist who is curious about advances and approaches in related areas but does not expect to expand his knowledge extensively. It would, perhaps, be of more value to the novice not yet committed, who needs a wide selection from which to make his choice.

There is no uniformity of presentation of the chapters, whether regarding structure, typescript (the volume has been produced by 'rapid manuscript reproduction' which does not account for the number of typographical errors in the list of contents), or length. Some, therefore, contain much information, whereas others may be described as brusque. This, however, may relate to the specialisation of the reader. Most tend towards the style of the mini-review or overview, while few assume the format of the scientific paper. These inconsistencies serve to emphasise the lack of cohesion in the book.

Peter Davies

\section{Genetic Engineering-Principles and Methods}

Volume 7. Edited by J K Setlow and A Hollaender. (Pp 359; figures + tables. \$52.50.) New York: Plenum Press. 1985.

The seventh volume of the series 'Genetic Engineering-Principles and Methods' contains 15 chapters on a wide variety of biological topics. These range from mainly methodological contributions, such as use of DNA expression libraries, computer analysis of DNA sequences, SP6 RNA polymerase reactions, and gene transfer using retroviral vectors, to reviews on some applications of DNA technology, including DNA replication and transcription, plant molecular genetics, bacterial pathogenicity, cytomegaloviruses, gene therapy, bacterial catabolic plasmids, and DNA markers for human genetic disorders. The more practically orientated chapters contain much useful experimental detail and all of the contributions are well written and presented, with sufficient references given to allow particular points to be pursued further. As a whole, the book is a worthwhile 